\title{
A near-infrared catalogue of the Galactic novae in the VVV survey area ${ }^{\star}$
}

\author{
R. K. Saito ${ }^{1,2,3,4}$, D. Minniti ${ }^{1,3,5,6}$, R. Angeloni ${ }^{1,3,7}$, M. Catelan ${ }^{1,3}$, J. C. Beamin ${ }^{1,3}$, J. Borissova ${ }^{2,3}$, I. Dékány ${ }^{1,3}$, \\ E. Kerins ${ }^{8}$, R. Kurtev ${ }^{2}$, and R. E. Mennickent ${ }^{9}$ \\ 1 Departamento de Astronomía y Astrofísica, Pontificia Universidad Católica de Chile, Av. Vicuña Mackenna 4860, $782-0436$ Macul, \\ Santiago, Chile \\ e-mail: rsaito@astro.puc.cl \\ 2 Departamento de Física y Astronomía, Facultad de Ciencias, Universidad de Valparaíso, Ave. Gran Bretaña 1111, Playa Ancha, \\ Casilla 5030, Valparaíso, Chile \\ 3 The Milky Way Millennium Nucleus, Av. Vicuña Mackenna 4860, 782-0436 Macul, Santiago, Chile \\ ${ }^{4}$ Universidade Federal de Sergipe, Departamento de Física, Av. Marechal Rondon s/n, 49100-000, São Cristóvão, SE, Brazil \\ 5 Vatican Observatory, Vatican City State 00120, Italy \\ 6 Departamento de Ciencias Fisicas, Universidad Andres Bello, Av. Republica 252, Santiago, Chile \\ 7 Department of Electrical Engineering, Center for Astro-Engineering, Pontificia Universidad Católica de Chile, \\ Av. Vicuña Mackenna 4860, 782-0436 Macul, Santiago, Chile \\ 8 Jodrell Bank Centre for Astrophysics, University of Manchester, Oxford Road, Manchester M13 9PL, UK \\ 9 Departamento de Astronomía, Universidad de Concepción, Casilla 160-C, Concepción, Chile
}

Received 7 February 2013 / Accepted 5 April 2013

\section{ABSTRACT}

\begin{abstract}
Context. Near-infrared data on classical novae contain useful information about the ejected gas mass and the thermal emission by dust formed during eruption, and provide independent methods to classify the objects according to the colour of their progenitors, and the fading rate and features seen after eruption. The VISTA Variables in the Vía Láctea survey (VVV) is a near-IR ESO Public Survey mapping the Milky Way bulge and southern plane. Data taken during 2010-2011 covered the entire area in the $J H K_{\mathrm{s}}$ bands plus some epochs in $K_{\mathrm{s}}$-band of the ongoing VVV variability campaign.

Aims. We used the VVV data to create a near-IR catalogue of the known Galactic novae in the 562 sq. deg. area covered by VVV. We also compiled the information about novae from the variability tables of the VVV variability campaign.

Methods. We used the novae list provided by VSX/AAVSO catalogue to search for all objects within the VVV area. From the 140 novae, we were able to retrieve the $J H K_{\mathrm{s}}$ colours of 93 objects. We also checked in the ongoing VVV variability campaign for the light curves of novae that erupted in the last years.

Results. The VVV near-IR catalogue of novae contains $J H K_{\mathrm{s}}$ photometry of 93 objects completed as of December 2012. VVV allows to monitor objects within up to $\Delta K_{\mathrm{s}} \sim 10$ mag range. VVV images can also be used to discover and study novae by searching for the expanding shell. Since objects are seen at different distances and reddening levels, the colour-magnitude and colour-colour diagrams show the novae spread in magnitude as well as in colour. Dereddened colours and reddening-free indices were used with caution and cannot be a good approach in all cases since the distance and spectral features prevent more conclusive results for some extreme objects. Light curves for some recent novae are presented.

Conclusions. Thanks to its high spatial resolution in the near IR and wide $K_{\mathrm{s}}$-range, the VVV survey can be a major contributor to the search for and study of novae in the most crowded and high-extinction regions of the Milky Way. The VVV survey area contains $\sim 35 \%$ of all known novae in the Galaxy.
\end{abstract}

Key words. novae, cataclysmic variables - Galaxy: stellar content - catalogs - surveys

\section{Introduction}

Cataclysmic variable stars (CVs) are close binary systems composed of a late-type star that transfers material to a more massive white dwarf (WD) companion. The typical mass transfer rates in CVs are $10^{-8}-10^{-11} M_{\odot} \mathrm{yr}^{-1}$, and the progressive accretion of hydrogen-rich material from the secondary star onto the hot surface of the WD can lead to a thermonuclear runaway, in which the accreted material is expelled from the system. This phenomenon is a so-called nova eruption (e.g., Frank et al. 2002; Warner 2003).

^ Based on observations taken within the ESO VISTA Public Survey VVV, Programme ID 179.B-2002.
During a nova eruption the object suddenly rises in brightness, typically becoming 8 to 15 mag brighter than its progenitor. The recurrence between nova outbursts is expected to be $10^{3}-10^{6}$ years, and depends on the mass of the white dwarf and the mass transfer rate. The systems are classified as classical novae when just one nova eruption is recorded. However, this is strongly biased by ancient records, which are limited to a few thousand years. Systems with two or more registered nova eruptions are classified as recurrent novae, in general associated with a high-mass transfer rate system onto a primary star with the mass close to the Chandrasekhar limit (Warner 2003). Since novae are usually found in eruption, the novae classification is in general based on the features seen during this phase, 


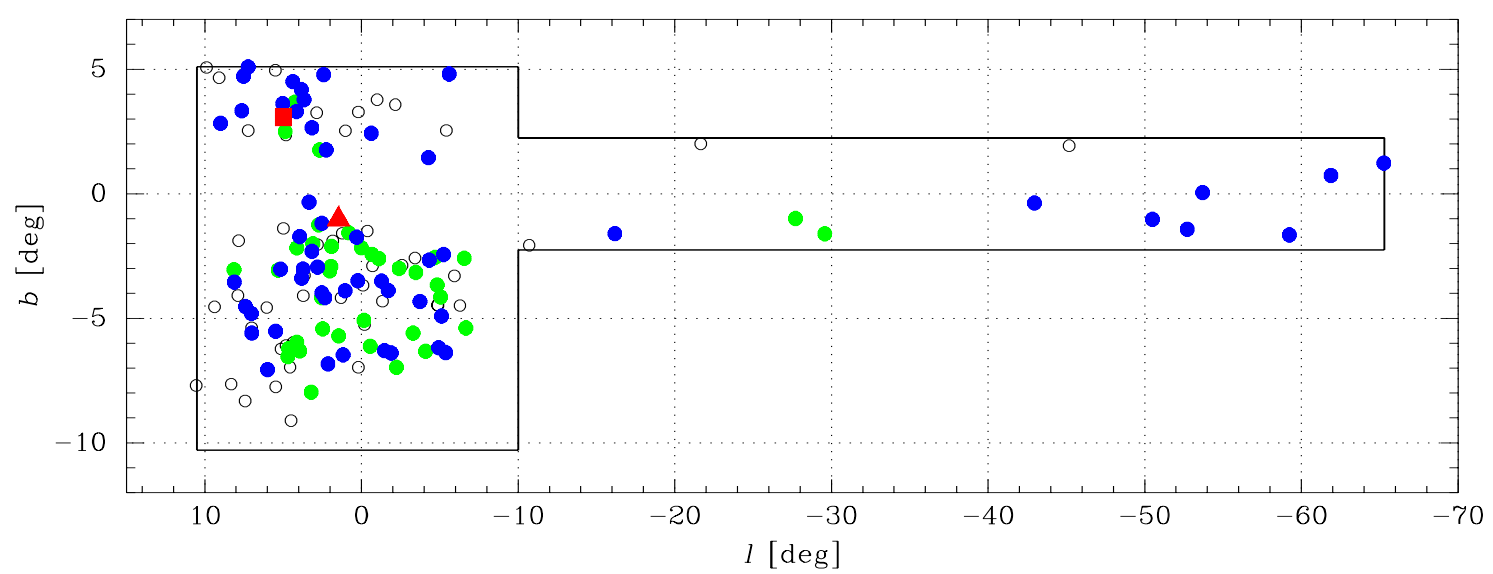

Fig. 1. Spatial distribution of all known Galactic novae in the VVV survey area. A total of 140 objects are present. Filled symbols mark the objects detected in the VVV data, while open symbols mark the novae with no detection in our data. The blue circles are novae with the coordinates matching a single source within $1^{\prime \prime}$ from the position given by the catalogue. Green circles mark the novae with doubtful photometry (see text). The red square is Nova Sgr 2012 progenitor, while the red triangle marks the position of Nova Sgr 2010b, caught during eruption. We note the presence of a "zone of avoidance" on the Galactic plane, with just a few objects belonging to the high-extinction regions of the MW.

such as the fading (speed class) rate after the nova eruption (e.g., Gaposchkin 1957) and the spectral differences (e.g., Williams 1992). More recently, alternative classifications have been proposed based on the Galactic component, with disk and bulge novae (e.g., della Valle \& Livio 1998), or that using automated selection and classification based on optical photometry (Darnley et al. 2004).

The infrared light can also contain the thermal emission by dust formed during the shell ejection. Models for thermonuclear runway predict the presence of material from the white dwarf in the ejecta, thus its spectroscopic analysis allows distinguishing between $\mathrm{CO}$ and ONe white dwarfs (Shafter et al. 2011). The importance of observing Galactic novae in the IR spectral region was fully understood when it was realized that they can represent a significant contributor to the interstellar medium (at least on local scales) of highly processed material, thus playing an active role in the chemical evolution of the Galaxy. Furthermore, IR observations provide independent methods for determining the ejected gas mass, a key parameter in every thermonuclear runaway model (Mason et al. 1998). Moreover, IR data can also help in the determination of both the global Galactic nova rate and the separate Galactic bulge and disk nova rates. Current Galactic nova samples, obtained mostly from optical surveys, are biased towards regions of lower optical extinction and therefore are incomplete within both the bulge and disk towards the Galactic plane. Previous determinations of the Galactic nova rate therefore vary by more than an order of magnitude, from 11 to $260 \mathrm{yr}^{-1}$ (Shafter 1997). The nova rate in M31 is better constrained from optical studies (e.g., Darnley et al. 2006), but it remains unclear whether the rates within both galaxies are consistent with fixed bulge and disk nova rates per unit galactic luminosity. Again, this uncertainty is due mostly to the poorly constrained knowledge of the Galactic rate. A large-scale nearinfrared time domain survey can allow novae to be identified throughout the Galactic bulge and disk and therefore facilitate a more complete determination of the nova rate within each stellar population.

The VISTA Variables in the Vía Láctea (VVV) is an ESO public near-IR survey scanning the Milky Way (MW) bulge and southern plane, in five near-IR bands $\left(Z Y J H K_{\mathrm{s}}\right)$, plus a variability campaign of about 100 epochs in the $K_{\mathrm{s}}$-band spanning many years (Minniti et al. 2010). VVV is about four magnitudes deeper than previous IR surveys, and thanks to its higher spatial resolution in the near-IR, enables deep observations in the most crowded and high-extinction regions of the Milky Way (e.g., Saito et al. 2012a).

Here we present a catalogue with the $J H K_{\mathrm{s}}$ colours of 93 novae in the VVV area. We discuss the colour properties and features seen in the near-IR. We also present the first results from the VVV variability campaign, including light curves for some novae. VVV can be a major contributor to the discovery and study of novae in the innermost regions of the Galaxy, objects that are beyond detection in the current novae searches.

\section{Observations}

VVV is an ESO Public Survey observing the MW bulge and inner southern plane in the near-IR with the VISTA Telescope located at Cerro Paranal Observatory in Chile (Minniti et al. 2010, and references therein). VVV observes an area of about $562 \mathrm{deg}^{2}$, within $-10.0^{\circ} \lesssim l \lesssim+10.5^{\circ}$ and $-10.3^{\circ} \lesssim$ $b \lesssim+5.1^{\circ}$ in the bulge, and within $294.7^{\circ} \lesssim l \lesssim 350.0^{\circ}$ and $-2.25^{\circ} \lesssim b \lesssim+2.25^{\circ}$ in the plane (see Fig. 1). The whole bulge and disk areas were fully observed in five near-IR passbands $\left(Z Y J H K_{\mathrm{s}}\right)$ during 2010-2011, while a variability campaign in the $K_{\mathrm{s}}$-band is ongoing, with $\sim 100$ pointings planned over many years (Saito et al. 2012a).

Photometric catalogues for the VVV observations are provided by the Cambridge Astronomical Survey Unit (CASU) ${ }^{1}$. The catalogues contain the positions, fluxes, and some shape measurements obtained from different apertures, with a flag indicating the most probable morphological classification. The VVV data are in the natural VISTA Vegamag system, with the photometric calibration in $J H K_{\mathrm{s}}$ performed using the VISTA magnitudes of unsaturated 2MASS stars present in the images. The single-band CASU catalogues for each tile were matched, creating a $J H K_{\mathrm{s}}$ catalogue of more than 173 million sources in the VVV bulge area (Saito et al. 2012b) and about 148 million sources in the disk. A similar procedure was adopted to match the $K_{\mathrm{s}}$-band catalogues from the variability campaign in order to create variability tables for selected fields.

1 http://casu.ast.cam.ac.uk/vistasp/ 


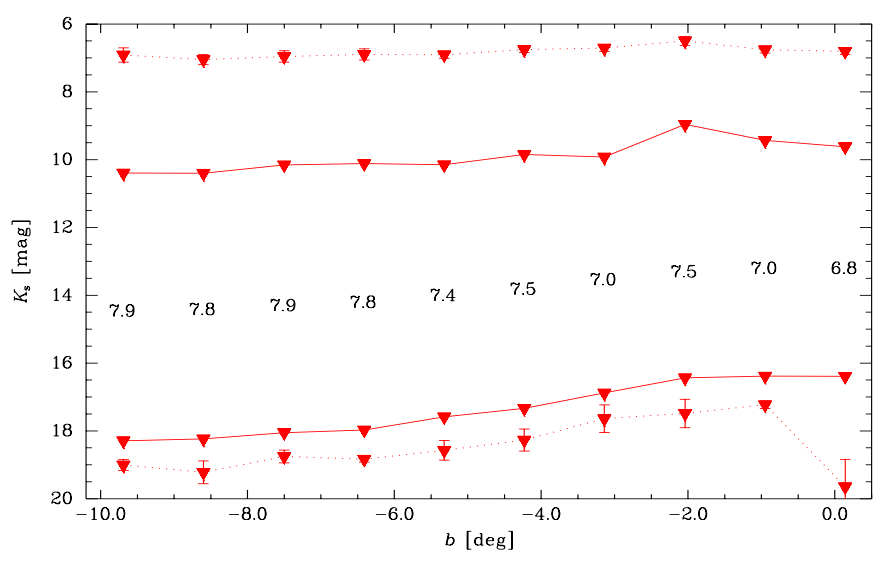

Fig. 2. Magnitude range covered by the VVV $K_{\mathrm{s}}$-band variability campaign observations along the Galactic minor axis. The solid lines with triangles mark the limits covered by the stellar sources, with the value of the total magnitude range shown in each position. The increasing sky brightness towards the Galactic centre is due to the contribution of the underlying, unresolved faint stars. The upper dotted line with triangles shows the mean value of the brightest sources, including objects flagged as saturated. Similarly, the lower dotted line with triangles marks the limiting magnitude of all sources, including those below the 5- $\sigma$ limit.

Different observing strategies between the bulge and disk areas, and effects caused by crowding and extinction, cause both the limiting magnitude and saturation to vary along the VVV area (Saito et al. 2012a,b). For instance, the $K_{\mathrm{s}}$-band limiting magnitude on the outer bulge is about $18.5 \mathrm{mag}$, while in the Galactic centre the limit is $K_{\mathrm{s}} \sim 16.5 \mathrm{mag}$ (see Fig. 2). The saturation limit varies in a shorter range. The $J H K_{\mathrm{s}}$ observations for a given field were taken in the same observational block $(\mathrm{OB})$, which guarantees quasi-simultaneous observations in the three bands. There is a time gap of only $190 \mathrm{~s}$ between each band. On the other hand, due to multiple-scheduling constraints on the side of the VISTA telescope operations, there is a little control on the cadence of the $K_{\mathrm{s}}$-band variability data within a given season, with the number of epochs planned in a given year being observed together with the other VISTA surveys according with the surveys requirements on the weather and visibility, for instance.

\section{The VVV novae catalogue}

Our novae list was taken from the International Variable Star indeX (VSX), provided by the American Association of Variable Star Observers ${ }^{2}$ (AAVSO). In this catalogue there are about 400 known novae in the Galaxy, 140 of which lie within the VVV area. Figure 1 shows their spatial distribution, with most objects concentrated in the Galactic bulge. We note that the information about some novae are from historical records or amateur astronomers, with the coordinates in general taken from observations while the objects were in eruption. Thus, to certify the coordinates with enough accuracy during the quiescent state, we checked for all objects in the SIMBAD database ${ }^{3}$. Finally, we defined the ICRS J2000 RA and DEC taken from SIMBAD as our coordinates, which were used as entries in our search for the VVV $J H K_{\mathrm{s}}$ photometry and composite colour images.

We defined as a valid match the cases where a source was found within 1 arcsec of the position given by the catalogue. This

\footnotetext{
2 http://www.aavso.org/vsx/index.php

simbad.u-strasbg.fr/simbad/
}

value agrees with the median values of the VVV image quality in the first season $\left(0{ }^{\prime} 89-0{ }^{\prime} 87\right.$, respective to $J$ and $K_{\mathrm{s}}$ observations) and the typical astrometric accuracy of the photometric catalogues ( $\lesssim 0$ '.5; Saito et al. 2012a). A complementary visual inspection of the images was performed for all novae candidates, in order to certify the presence (or absence) of a target in the given position. In some cases the source was rejected because of the presence of a faint object in a closer position to the coordinate, seen during the visual inspection of the images, but beyond the detection limit of the CASU aperture photometry, used to build the VVV photometric catalogues (see Sect. 2). In other cases multiple sources are seen in a similarly close distance from the entry coordinates. In this procedure we secured the information for 55 novae within $\leq 1$ arcsec from the catalogue position, with 27 of them within the VIRCAM pixel scale of 0 '.34 pixel $^{-1}$. A complementary search included objects matching the position given by the catalogue within 2 arcsec. By this procedure we retrieved the photometry of another 37 objects. These are all fainter than $K_{\mathrm{s}} \sim 13 \mathrm{mag}$ and were classified as doubtful sources. We note that the source confusion in the most crowded regions of Galactic plane and bulge means that a search based only on the coordinates does not guarantee that in all cases the source found in a closer position to the coordinates is in fact the nova remnant. Thus these should be seen as candidate for near-IR counterparts of the novae remnant.

Therefore, from the 140 novae within the VVV area, we are able to provide the $J H K_{\mathrm{s}}$ colours of 93 objects: 55 classified as valid matches (27 within the pixel scale) and 37 as doubtful sources. The other objects are beyond our detection limit (progenitors in quiescence with $K_{\mathrm{s}} \gtrsim 18 \mathrm{mag}$ ), or have coordinates not accurate enough to allow us to identify the target in the field. Table 1 presents the $J H K_{\mathrm{s}}$ photometry of all novae detected in the VVV area, while in the Appendix we provide the composite $J H K_{\mathrm{s}}$ finding charts for all objects.

Since the VVV $J H K_{\mathrm{s}}$ observations were performed during 2010-2011, we are also able to provide the information about the progenitors of the most recent novae, as well as the fading curve after the nova eruption (see Sect. 5). Interestingly, the observations of Nova Sgr 2010b were secured while the object was in eruption (Saito \& Minniti 2012a). Complementary information, including the timings of the VVV colour and variability campaign observations for all novae erupted in this century, is presented in Table 2 .

\section{The near-IR colours}

Figure 3 shows the $K_{\mathrm{s}}$ vs. $\left(J-K_{\mathrm{s}}\right)$ colour-magnitude diagram (CMD) and the $(J-H)$ vs. $\left(H-K_{\mathrm{s}}\right)$ colour-colour diagram (CCD) in the top panels for all novae in the VVV area. We used different symbols to denote the objects classified as valid matches and the doubtful ones, as described in the previous section. The reddening vector associated with an extinction of $E(B-V)=1$, based on the relative extinctions of the VISTA filters, and assuming the Cardelli et al. (1989) extinction law, is also shown.

The Galactic novae range in magnitude and colour, with all objects appearing red, with $\left(J-K_{\mathrm{s}}\right)>0$ (the only exception is Nova Cir 1906, with $\left.\left(J-K_{\mathrm{s}}\right)=-0.228\right)$. Interestingly, a large fraction of novae were beyond the detection limit of previous near-IR surveys (e.g., 2MASS, DENIS; Skrutskie et al. 2006; Epchtein et al. 1994), thus we are now reporting their IR-colours for the first time in a homogeneous data set. We note that the novae are spread across the whole VVV area (see Fig. 1), and therefore are affected by different extinction. For instance, in the 


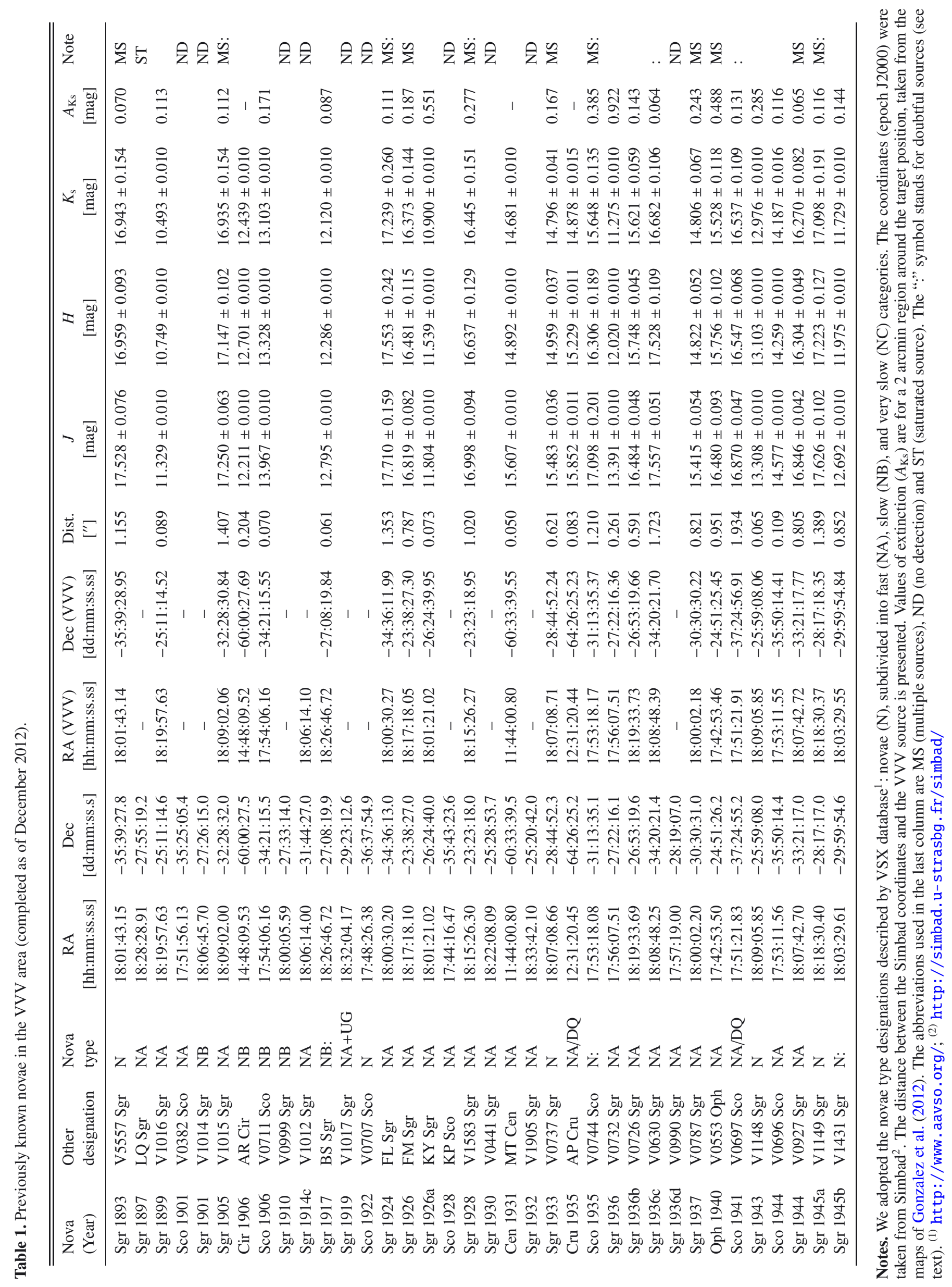


R. K. Saito et al.: A near-IR catalogue of the Galactic novae in the VVV survey area

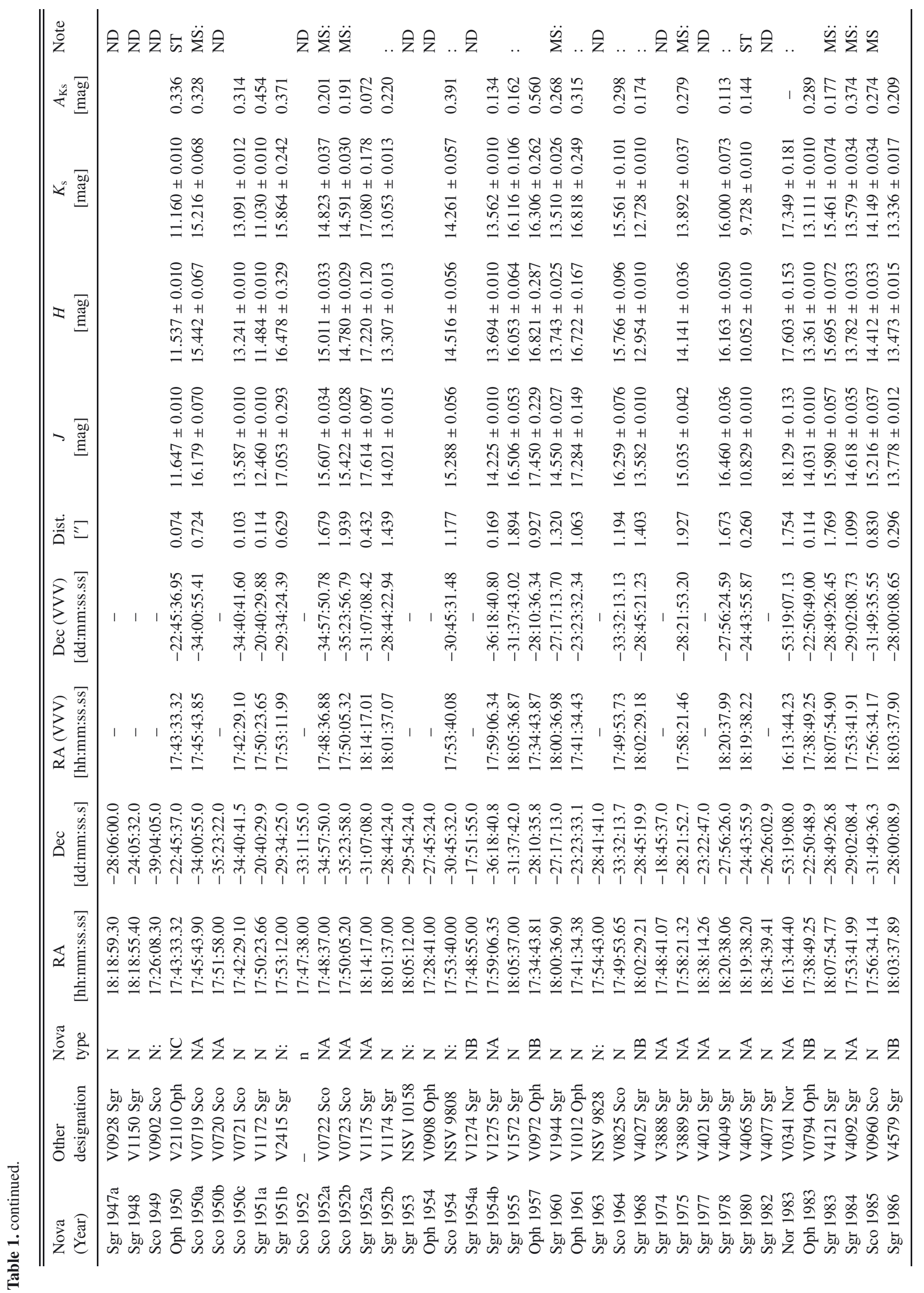


A\&A 554, A123 (2013)




R. K. Saito et al.: A near-IR catalogue of the Galactic novae in the VVV survey area

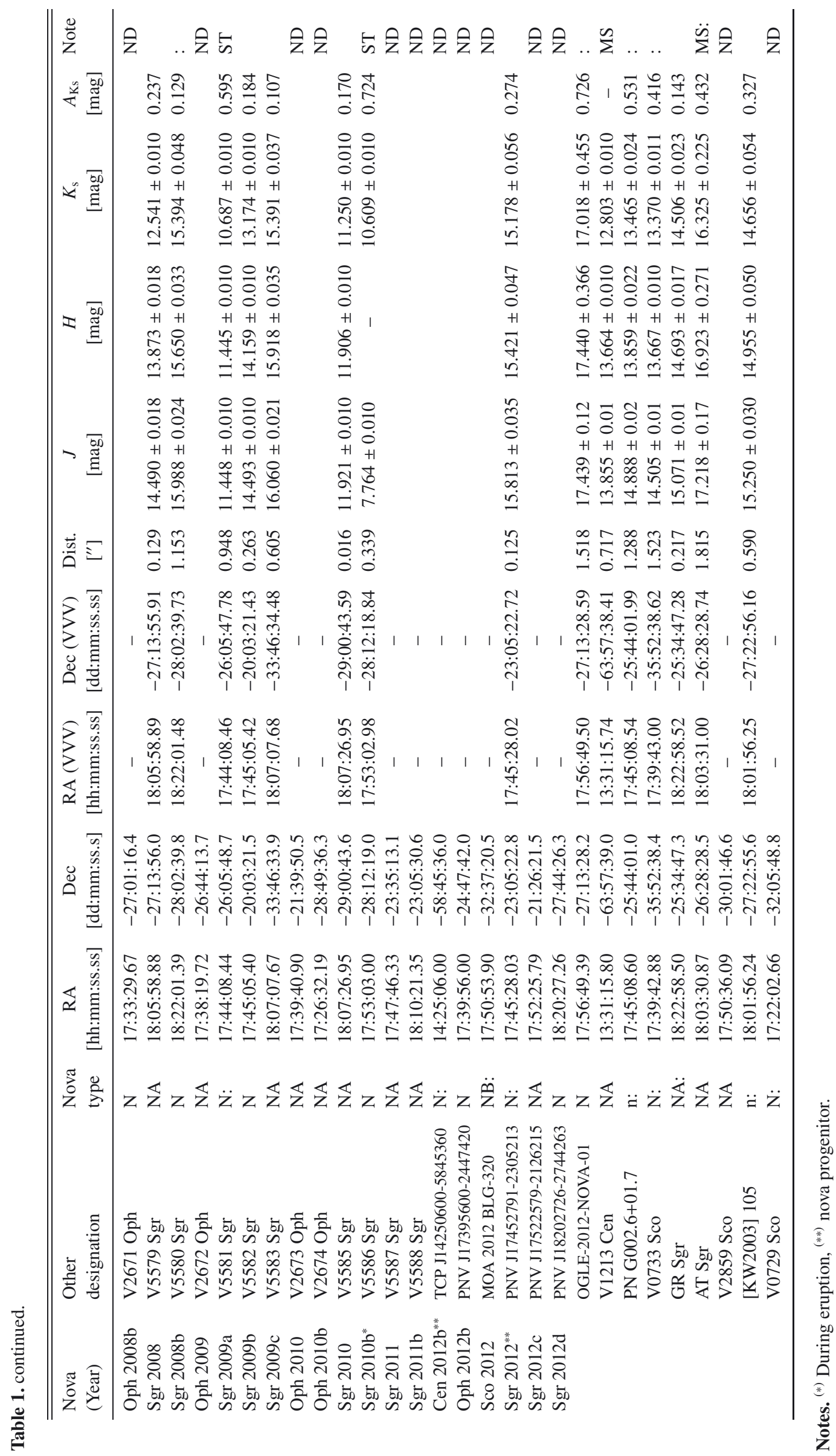

A123, page 7 of 20 
A\&A 554, A123 (2013)

Table 2. Novae eruptions in the VVV area from January 2001 to the December 2012.

\begin{tabular}{|c|c|c|c|c|c|}
\hline Nova & $\begin{array}{c}\text { Other } \\
\text { designation }\end{array}$ & $\begin{array}{l}\text { Eruption } \\
\text { date }\end{array}$ & $\begin{array}{l}\text { VVV } J H K_{\mathrm{s}} \\
\text { observation }\end{array}$ & $\begin{array}{l}\text { First } K_{\mathrm{s}} \text {-band epoch } \\
\text { (variability campaign) }\end{array}$ & Note \\
\hline Sco 2001 & V1178 Sco & 2001 & 20100810 & 20100912 & Remnant \\
\hline Sgr 2001 & V4643 Sgr & 2001 & 20100409 & 20110806 & Remnant \\
\hline Sgr 2001b & V4739 Sgr & 2001 & 20100814 & 20101015 & Remnant \\
\hline Sgr 2001c & V4740 Sgr & 2001 & 20100815 & 20110805 & Remnant \\
\hline Sgr 2002 & V4741 Sgr & 2002 & 20100810 & 20100912 & Remnant \\
\hline Sgr 2002b & V4742 Sgr & 2002 & 20100328 & 20100330 & Remnant \\
\hline Sgr $2002 d$ & V4744 Sgr & 2002 & 20100318 & 20100929 & Remnant \\
\hline Sgr 2003b & V5113 Sgr & 2003 & 20100328 & 20100826 & Remnant \\
\hline Oph 2004 & V2574 Oph & 2004 & 20100330 & 20100410 & Remnant \\
\hline Sco 2004 & V1186 Sco & 2004 & 20100803 & 20100826 & Remnant \\
\hline Sco $2004 b$ & V1187 Sco & 2004 & 20100803 & 20100826 & Remnant \\
\hline Sgr 2004 & V5114 Sgr & 2004 & 20100815 & 20101024 & Remnant \\
\hline Cen 2005 & V1047 Cen & 2005 & 20100307 & 20100329 & Remnant \\
\hline Nor 2005 & V0382 Nor & 2005 & 20100306 & 20100306 & Remnant \\
\hline Sco 2005 & V1188 Sco & 2005 & 20101001 & 20110727 & Remnant \\
\hline Sgr 2005a & V5115 Sgr & 2005 & 20100328 & 20100330 & Remnant \\
\hline Sgr $2005 b$ & V5116 Sgr & 2005 & 20100815 & 20101015 & Remnant \\
\hline Oph 2006 & V2575 Oph & 2006 & 20100330 & 20100409 & Remnant \\
\hline Sgr 2006 & V5117 Sgr & 2006 & 20100423 & 20101025 & Remnant \\
\hline Oph 2007 & V2615 Oph & 2007 & 20100317 & 20110612 & Remnant \\
\hline Nor 2007 & V0390 Nor & 2007 & 20100316 & 20100815 & Remnant \\
\hline Oph 2008a & V2670 Oph & 2008 & 20100317 & 20110612 & Remnant \\
\hline Oph 2008b & V2671 Oph & 2008 & 20110507 & 20110613 & Remnant \\
\hline Sgr 2008 & V5579 Sgr & 2008 & 20100412 & 20110805 & Remnant \\
\hline Sgr $2008 b$ & V5580 Sgr & 2008 & 20100408 & 20100827 & Remnant \\
\hline Oph 2009 & V2672 Oph & 2009 & 20100413 & 20110613 & Remnant \\
\hline Sgr 2009a & V5581 Sgr & 2009 & 20100330 & 20100330 & Remnant \\
\hline Sgr $2009 b$ & V5582 Sgr & 2009 & 20100410 & 20110614 & Remnant \\
\hline Sgr 2009c & V5583 Sgr & 2009 & 20100423 & 20100818 & Remnant \\
\hline Oph 2010 & V2673 Oph & $20100115^{a}$ & 20100410 & 20110614 & Remnant \\
\hline Sgr 2010 & V5585 Sgr & $20100120^{b}$ & 20100423 & 20100826 & Remnant \\
\hline Oph 2010b & V2674 Oph & $20100218^{c}$ & 20110509 & 20110726 & Remnant \\
\hline Sgr 2010b & V5586 Sgr & $20100423.782^{d}$ & $\begin{array}{c}20100423.2457(H) \\
20100423.2479\left(K_{\mathrm{s}}\right) \\
20100423.2501(J)\end{array}$ & 20100912 & During eruption \\
\hline Sgr 2011 & V5587 Sgr & $20110125^{e}$ & 20100318 & 20100929 & Progenitor \\
\hline Sgr 2011b & V5588 Sgr & $20110407^{f}$ & 20100421 & 20101006 & Progenitor \\
\hline Cen $2012 b$ & TCP J14250600-5845360 & $20120405^{g}$ & 20100327 & 20100309 & Progenitor \\
\hline Sgr 2012 & PNV J17452791-2305213 & $20120421^{h}$ & 20100318 & 20100929 & Progenitor \\
\hline Oph 2012b & PNV J17395600-2447420 & $20120519^{i}$ & 20100413 & 20110612 & Progenitor \\
\hline Sco 2012 & MOA 2012 BLG-320 & $20120522^{j}$ & 20100829 & 20100925 & Progenitor \\
\hline Sgr 2012c & PNV J17522579-2126215 & $20120626^{k}$ & 20100325 & 20100929 & Progenitor \\
\hline \multirow[t]{2}{*}{ Sgr 2012d } & PNV J18202726-2744263 & $20120712^{l}$ & 20100408 & 20100827 & Progenitor \\
\hline & OGLE-2012-NOVA-01 & $20120502^{m}$ & 20100328 & 20100826 & Progenitor \\
\hline
\end{tabular}

References. ${ }^{(a)}$ Nakano et al. (2010a); ${ }^{(b)}$ Nishiyama et al. (2010); ${ }^{(c)}$ Nakano et al. (2010b); ${ }^{(d)}$ Kiyota et al. (2010); ${ }^{(e)}$ Nakano et al. (2011); (f) Nishiyama et al. (2011); ${ }^{(g)}$ Green (2012a); ${ }^{(h)}$ Green (2012b); ${ }^{(i)}$ Walter \& Buil (2012); ${ }^{(j)}$ Waagen (2012); ${ }^{(k)}$ Yamaoka \& Itagaki (2012); ${ }^{(l)}$ Nishimura \& Nakano (2012); and ${ }^{(m)}$ Kozlowski et al. (2012).

bulge area where most novae are located, reddening spans from $E(B-V) \lesssim 0.2$ at $b \sim-10^{\circ}$ to $E(B-V) \sim 10$ closer to the Galactic centre (e.g., Gonzalez et al. 2012).

In the $(J-H)$ vs. $\left(H-K_{\mathrm{s}}\right) \mathrm{CCD}$ the objects are seen to be more concentrated at low values of $\left(H-K_{\mathrm{s}}\right)$, but spread along $(J-H)$ by more than 1 mag. The colours of main sequence stars (with spectral type from B0 to M4, adapted from
Ducati et al. 2001) are presented in the CMD in the case of $A_{K \mathrm{~s}}=0 \mathrm{mag}$ (no extinction) and when applying the median value of extinction among our targets of $A_{K \mathrm{~s}}=0.243 \mathrm{mag}$ (corresponding to $E(B-V) \sim 0.67 \mathrm{mag})$. The comparison reveals that part of our sources coincide in colour with the main sequence stars and therefore suggests that some of our novae candidates can actually be old field stars, which match a position 
R. K. Saito et al.: A near-IR catalogue of the Galactic novae in the VVV survey area
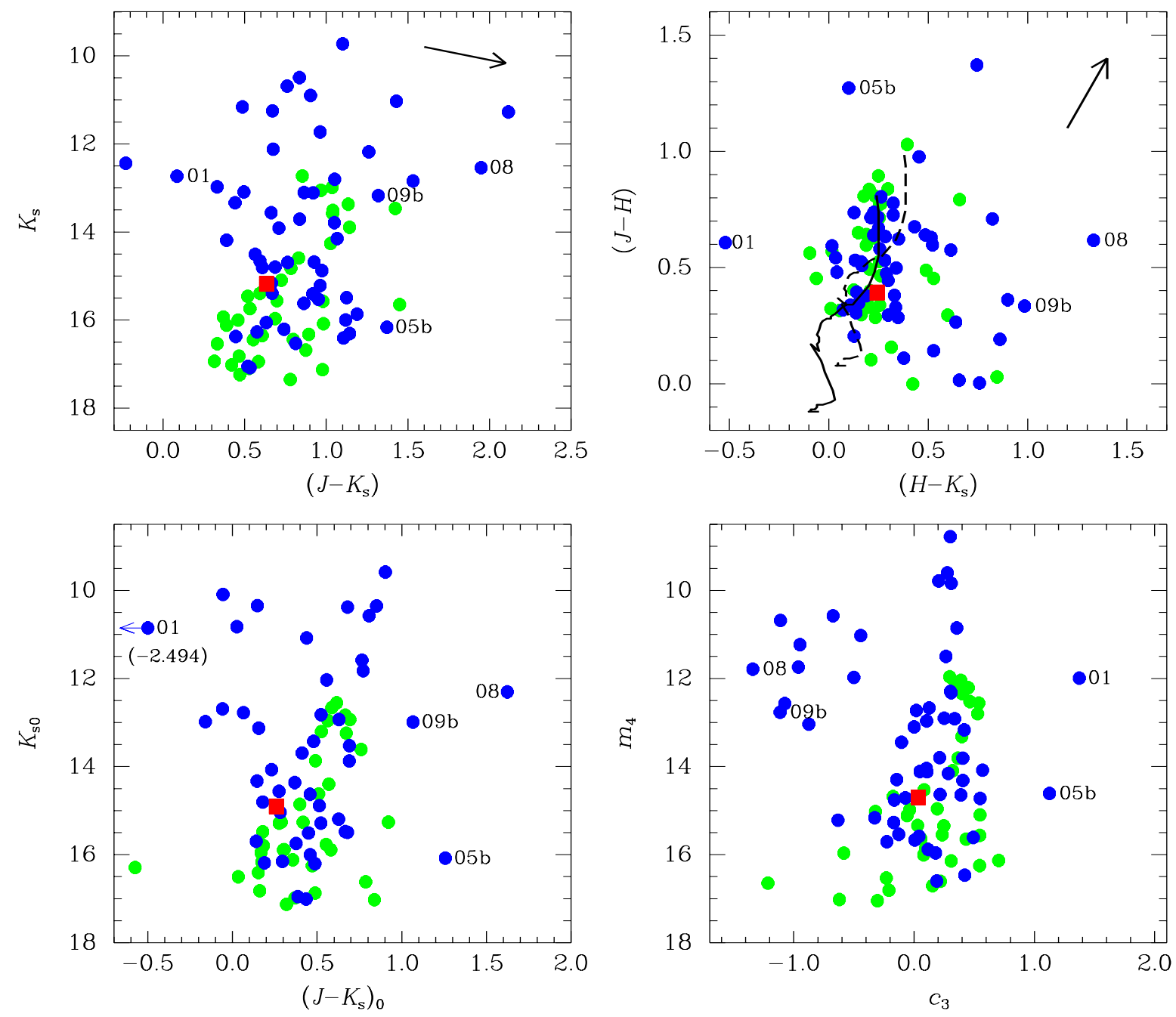

Fig. 3. Top-left panel: $K_{\mathrm{s}}$ vs. $\left(J-K_{\mathrm{s}}\right)$ CMD for all novae detected in the VVV area. The colour pattern and symbols are the same as used in Fig. 1. Top-right panel: the corresponding $(J-H)$ vs. $\left(H-K_{\mathrm{s}}\right)$ colour-colour diagram. Bottom-left: dereddened CMD for all bulge novae, using the reddening maps of Gonzalez et al. (2012) and assuming the Cardelli et al. (1989) extinction law (no disk novae are plotted). Bottom right: CMD using the reddening-free parameters provided by Catelan et al. (2011). The colour of main sequence stars is shown in the $(J-H)$ vs. $\left(H-K_{\mathrm{s}}\right)$ CCD in the case of $A_{K \mathrm{~s}}=0 \mathrm{mag}$ (no extinction, solid line) and for $A_{K \mathrm{~s}}=0.243$ (the median value of extinction among our targets, shown as a dashed line; adapted from Ducati et al. 2001). The reddening vector associated with an extinction of $E(B-V)=1$, based on the relative extinctions of the VISTA filters, and assuming the Cardelli et al. (1989) extinction law, is also shown in the top panels. Four objects are labelled in the plots: Nova Sgr 2001, Nova Sgr 2005b, Nova Sgr 2008, and Nova Sgr 2009b.

close to the novae coordinates and suffer from reddening at different levels. Individual values of $A_{K \mathrm{~s}}$ for all bulge novae are listed in Table 1. Although the Ducati et al. (2001) data are in the JHK system, it is close enough to the VISTA system to be is useful for the comparison. Four objects are seen with the most extreme colours: Nova Sgr 2001 and Nova Sgr 2005b, with the largest $(J-H)$ but low $\left(H-K_{\mathrm{s}}\right)$; and Nova Sgr 2008 and Nova Sgr 2009b, showing the highest $\left(H-K_{\mathrm{s}}\right)$ values at relatively low values of $(J-H)$. The complementary $J H K_{\mathrm{s}}$ spectral energy distributions (SED) for these selected objects are shown in Fig. 4. While Nova Sgr 2001 and Nova Sgr 2005b show a maximum close to the $H$-band, the SED of Nova Sgr 2008 and Nova Sgr 2009b reveal a monotonic increase in flux towards longer wavelengths.

To minimize the reddening effects we calculated the dereddened $J_{0}, H_{0}$, and $K_{\mathrm{s} 0}$ colours for the bulge novae, using the maps of Gonzalez et al. (2012) and the relative extinctions of the VISTA filters and assuming the Cardelli et al. (1989) extinction law. A dereddened $K_{\mathrm{s} 0}$ vs. $\left(J-K_{\mathrm{s}}\right)_{0}$ CMD is presented in the bottom-left panel of Fig. 3. Since the Gonzalez et al. (2012) maps do not cover the disk area we prefer to exclude the few novae located in this region from the plot, instead of using a different prescription for the extinction in the disk, which does not guarantee good agreement with the procedure used in the bulge area (e.g., Saito et al. 2012a). We also note that the reddening law is known to change close to the Galactic centre (e.g., Nishiyama et al. 2009), but in our sample just one object (Nova Sgr 2001) belongs to this region.

A set of reddening-free indices provided by Catelan et al. (2011), based on the extinction law of Rieke \& Lebofsky (1985) for the VISTA filters, was also computed. The $m_{4}$ pseudo-magnitude and the $c_{3}$ pseudo-colour are defined as

$$
\begin{aligned}
m_{4} & \equiv K_{\mathrm{s}}-1.22(J-H), \\
c_{3} & \equiv(J-H)-1.47\left(H-K_{\mathrm{s}}\right) .
\end{aligned}
$$

The $m_{4}$ vs. $c_{3}$ CMD is shown in the bottom-right panel of Fig. 3. We note the indices shown in the equations depend on the 
effective wavelengths of each filter, assumed to be a flat distribution here, which is certainly different from the near-IR spectra of novae. Refined indices based on actual near-IR spectra covering different luminosity classes and spectral types are currently being computed, and quantitative tests in the case of actual near-IR spectra of novae will also be carried out in the future.

One important aspect seen in all panels of Fig. 3 is the absence of a clear-cut correlations or trends, even using dereddened colours and reddening-free indices, the resulting distributions do not appear to have straightforward interpretations. The differences in colour arise mostly because (i) the objects are seen at different distances and positions in the Galaxy, and thus can suffer from extinction and reddening at different levels compared to the $2 \mathrm{D}$ reddening maps of (Gonzalez et al. 2012); and (ii) the spectral energy distribution in the near-IR can be affected by emission lines and thermal emission by a dust shell produced during eruption. The latter are time-dependent and mostly valid for recent novae. Depending on the evolution of the dust shells created in the outbursts, a variety of emission lines are seen in early post-outburst near-IR spectra. Moreover, for older novae the peak emission from the dust shells is shifted to longer wavelengths (e.g., Venturini et al. 2002; Banerjee \& Ashok 2012). Four objects seen with the extreme colours in the plots of Fig. 3 are discussed in more detail in the following, namely Nova Sgr 2001, Nova Sgr 2005b, Nova Sgr 2008, and Nova Sgr 2009 b.

Ashok et al. (2006) classify Nova Sgr 2001 as an "NA" fast novae, with absence of dust formation after the eruption. Its near-IR spectrum evolves significantly from the early decline stage (March 2001) to the coronal phase (August 2001, Ashok et al. 2006). Nova Sgr 2001 is the innermost known nova in the Galaxy, with coordinates $(l, b)=(3.345,-0.337) \mathrm{deg}$, with an extinction of $A_{K \mathrm{~s}}=1.88 \mathrm{mag}$ according to the maps of Gonzalez et al. (2012) using Nishiyama et al. (2009) extinction law, equivalent to $A_{V} \simeq 15.9 \mathrm{mag}$.

Our data taken during the quiescent phase (on April 2010) show the remnant of Nova Sgr 2001 as a relatively blue object, with the maximum intensity in the near-IR around the $H$-band (see Fig. 4). However, data taken with UKIRT on March 2012 show Nova Sgr 2001 with a flatter SED in the near-IR, with the maximum intensity towards longer wavelengths (Varricatt et al. 2012).

Ashok et al. (2006) estimated a distance to the object of $d=3.3 \mathrm{kpc}$. Thus, since our calculations using Gonzalez et al. (2012) maps assume the total extinction in the line of sight, it could not be a good approach to estimating the reddening for nearby objects because it overestimates the corrections. In this case, three-dimensional extinction maps are necessary, in order to estimate the relative extinction at given distance (e.g., Chen et al. 2013). On the other hand, the $c_{3}$ colour seems to be dominated by the $H$-band (by construction, see Eq. (2)), thus the presence of features, such as emission lines or simply the slope of the SED, contribute to shifting the value of the $c_{3}$ pseudo-colour from a relatively low to a relatively high value, depending on whether the emission peaks around the $H$-band or not. This explains why Nova Sgr 2001 and Nova Sgr 2005b, whose spectra do peak around $H$ (Fig. 4), have high positive $c_{3}$ values, whereas the remaining novae, whose spectra monotonically increase towards longer wavelengths, have - on the contrary - high negative $c_{3}$ values.

Nova Sgr 2005b is similar in colour with Nova Sgr 2001. Its remnant is a Supersoft X-ray variable source, presenting an orbital period of $P_{\text {orb }}=2.97 \mathrm{~h}$ (Dobrotka et al. 2008; Sala et al. 2008, 2010), which puts the object on the edge of the 2-3 h CVs period gap (e.g., Warner 2003). The object is located beyond

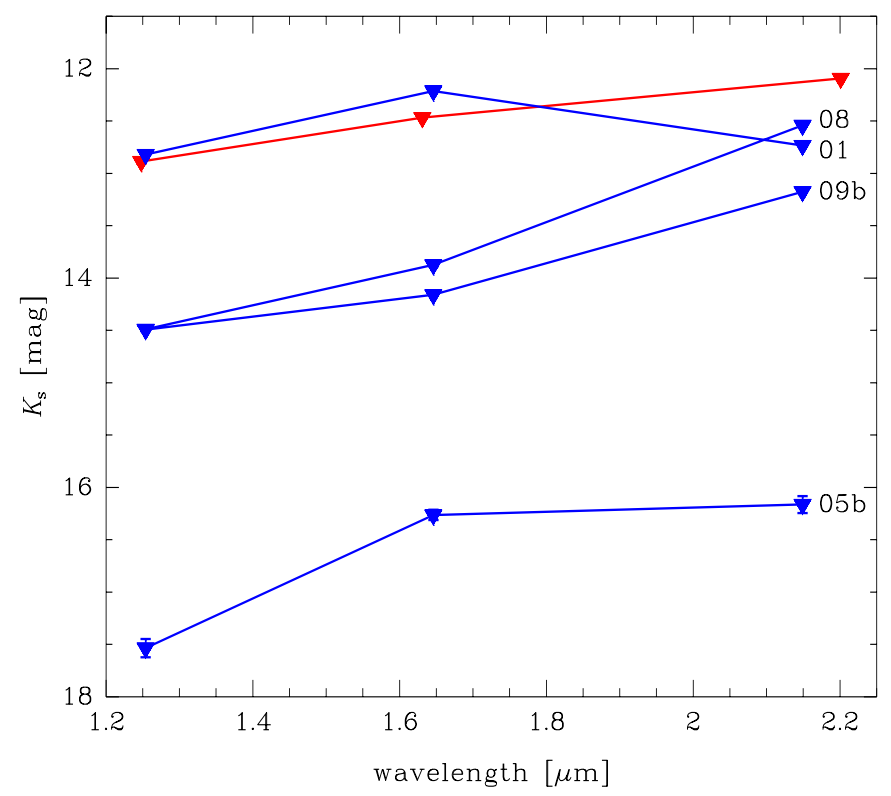

Fig. 4. Spectral energy distribution for the novae showing the extreme colours in Fig. 3: Nova Sgr 2001, Nova Sgr 2005b, Nova Sgr 2008, and Nova Sgr 2009b. Data for Nova Sgr 2001 taken with UKIRT are also shown (red points; see Sect. 4).

the bulge, with $(l, b)=(2.136,-6.832)$ deg and a distance of $d=11 \pm 3 \mathrm{kpc}$ estimated by Sala et al. (2008). Nova Sgr $2005 \mathrm{~b}$ lies in the lower part of the CMDs, with $K_{\mathrm{s}}=16.163$ and $K_{\mathrm{s}, 0}=16.079$. The presence of such distant objects in our data demonstrates our capability to monitor even the most distant novae in the Galaxy.

In the other extreme are Nova Sgr 2008 and 2009b. Nova Sgr 2008 was discovered in eruption on 2008 April 18 (Nakano et al. 2008). It is a nearby fast Fe II nova, located at $(l, b)=$ (3.734, -3.022) deg at distance of $d=4.4 \pm 0.2 \mathrm{kpc}$ (Russell et al. 2008; Raj et al. 2011). The very red colours even in $\left(J-K_{\mathrm{S}}\right)_{0}$ are related to the presence of dust. Spectra taken in the late stages after the eruption indicate dust formation during the nova remnant's development (Raj et al. 2011). Nova Sgr 2009b (Sun \& Gao 2009) is located in a low-extinction region with $(l, b)=(7.531,4.719) \mathrm{deg}$ and $A_{K_{\mathrm{s}}}=0.1845 \mathrm{mag}$. There are no entries in the literature estimating its distance or classifying the nova remnant. However, by comparing their colours, one can infer that Nova Sgr 2009b was similar to the Nova Sgr 2008 remnant. In this case, Nova Sgr 2009b could be surrounded by dust that is expelled from the system after the eruption.

\section{The VVV variability campaign on novae}

The IR photometric monitoring of several novae outbursts has already in the 1990's allowed two fundamentally different types of objects to be distinguished, usually referred to as $\mathrm{CO}$ and ONe novae. While the first seems to arise from thermonuclear runaway on a relatively low-mass WD and tends to form considerable amounts of dust at later stages, the latter turn out to be associated with massive $\left(\geq 1.2 M_{\odot}\right)$ WD that have outbursts that exhibit a coronal line emission phase with little or no dust production.

Particularly useful in identifying extreme $\mathrm{CO}$ and $\mathrm{ONe}$ novae are the $K_{\mathrm{s}}$-band light curves: because of the thermal emission from dust during the condensation phase, $\mathrm{CO}$ novae reach an emission peak within a few months of the optical maximum, 


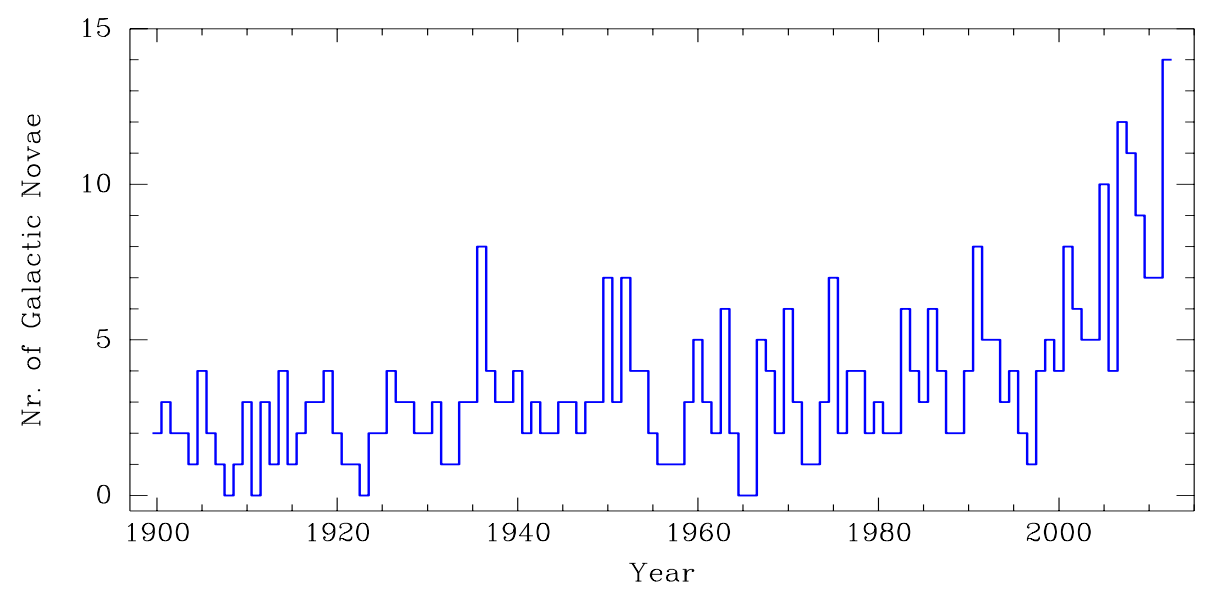

Fig. 5. Historical record of all classical novae discovered in the MW since year 1900. Despite the increment in the nova rate seen in the past two decades, the nova rate is still below what is seen in nearby galaxies. The data we are completed as of December 2012. declining exponentially thereafter as a consequence of the decreasing density of the shell owing to expansion (emblematic is the case of NQ Vul; Sato et al. 1978). ONe novae, in contrast, decline rapidly shortly after the optical maximum and then have a positive change in the slope rate (e.g., V1974 Cyg; Woodward et al. 1997) because of the increasing contribution of several IR forbidden emission lines (e.g., [Ca VIII] $2.32 \mu \mathrm{m}$, [Si VII] $2.47 \mu \mathrm{m}$ ), whose intensity strengthens during the coronal phase. It acts thus as the effective coolant of the ejecta (e.g., as in QU Vul; Greenhouse et al. 1988).

The VVV Survey, for the first time, will provide wellsampled light curves in the $K_{\mathrm{s}}$-band for about 100 Galactic novae that exploded in the last $\sim 150$ years in the Galactic bulge and in the southern part of the Galactic disk. At the time of writing, the VVV variability campaign is going on, and in the next few years until the completion of the survey (2016 or later), we will be able to finally build a coherent picture of nova IR behaviour, at quiescence, as well as to discover new novae in highly extincted regions that are missed in the optical.

There are about 400 known novae in the Milky Way, but the comparison with the nova rate in nearby galaxies shows that a number of novae are lost every year in the Galaxy. While the historical records show a rate below a dozen nova discovered per year in the MW (see Fig. 5), several results point to a nova rate spanning $\sim 30-40 \mathrm{yr}^{-1}$, or even higher reaching up to $\sim 260 \mathrm{yr}^{-1}$ (Liller \& Mayer 1987; Liller 1993; della Valle \& Livio 1994; Shafter 1997; Shafter \& Irby 2001).

Not coincidentally, the spatial distribution of novae in the bulge and southern plane shows a zone of avoidance (see Fig. 1), with just a few objects belonging to the high-extinction regions of the Galaxy. This arises because the current searches for novae are performed with small-aperture telescopes in the optical. The VVV Survey combines deep and high-spatial resolution observations in the near-infrared, allowing one to search for novae even in the most crowded and high-extinction regions of our Galaxy. Very illustrative is the recent discovery of VVV-NOV-001, the first nova candidate from the VVV data, located in a high-extinction region in the inner bulge with $(l, b)=$ (8.897, -0.158) deg and $A_{V} \sim 11 \mathrm{mag}$ (Saito et al. 2012c).

Figure 2 shows the magnitude range covered by VVV at different levels of crowding in the bulge. Even in the most problematic case, which is close to the Galactic centre, the VVV observations allow us to monitor over a range in magnitudes spanning from $\Delta K_{\mathrm{s}}>7 \mathrm{mag}$, reaching even $\Delta K_{\mathrm{s}} \sim 10 \mathrm{mag}$ when using data taken below 5- $\sigma$ accuracy and saturated sources. As of writing, we are able to provide light curves with a few points for

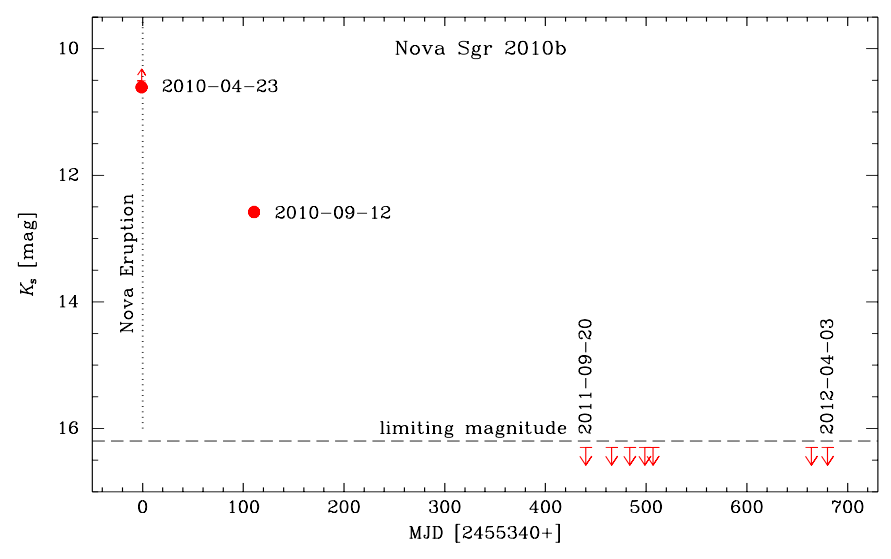

Fig. 6. VVV $K_{\mathrm{s}}$-band light curve of Nova Sgr 2010b. The VVV data caught the object about 0.5 days before its first detection in eruption. The object is seen as a saturated source on 23 March 2010 and recorded about 2 mags fainter on 12 Sep. 2010. Sgr 2010b is beyond detection $\left(K_{\mathrm{s}} \gtrsim 16.2 \mathrm{mag}\right.$ in the VVV $K_{\mathrm{s}}$-band aperture catalogues for this field) in a sequence of observations from Sep. 2011 to Apr. 2012.

some objects, which demonstrates our ability to monitor novae at all phases depending on the magnitude in each case. Figures 6-8 show VVV light curves of Nova Sgr 2010b (Saito \& Minniti 2012a), 2011, and 2012 (Saito \& Minniti 2012b), respectively.

These $K_{\mathrm{s}}$-band light curves at quiescence will allow us to study any kind of orbital effect that could be related to the influence on the secondary star by the accreting WD, and when combined with the IR colours, will also help quantify (via modelling of ellipsoidal variations of the secondary) fundamental parameters of the nova system, such as spectral type of the secondary, mass ratios, orbital periods, and inclinations (Mason et al. 1998).

\section{Perspectives on the search for novae using the VVV images}

There is a well-known discrepancy in the actual amount of mass ejected in a CN outburst as predicted by theory and numerical simulations on one hand, and as derived from infrared and radio observations, on the other - where the latter is systematically higher by up to a factor of about ten (Shaviv 2002; Shaviv \& Dotan 2012).

Even if there are strong caveats on the methods and assumptions used for determining the mass of the ejecta (e.g., Shara et al. 2010), the analysis of the most recent observational 


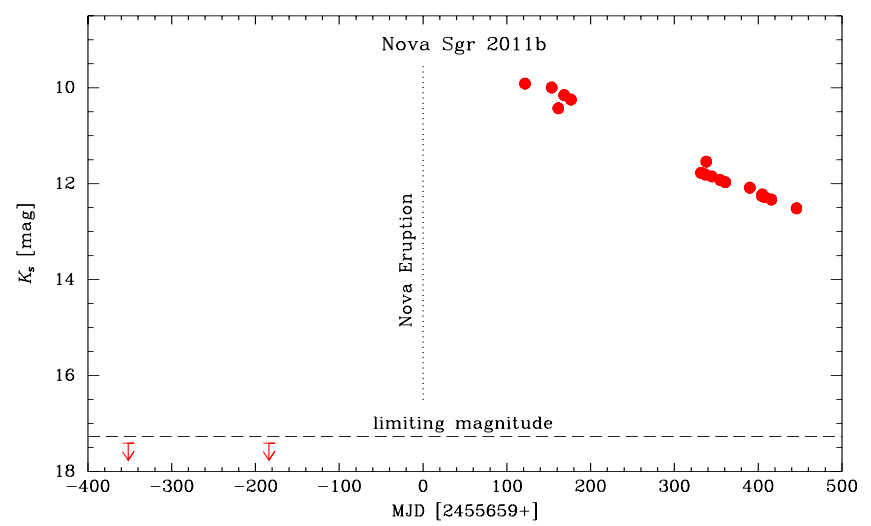

Fig. 7. VVV $K_{\mathrm{s}}$-band light curve of Nova Sgr 2011b. Several epochs starting on 21 Apr. 2011 registered the object fading after its eruption on 07 Apr. 2011. The nova progenitor is beyond the limiting magnitude in two observations taken during $2010\left(K_{\mathrm{s}} \gtrsim 17.3 \mathrm{mag}\right)$.

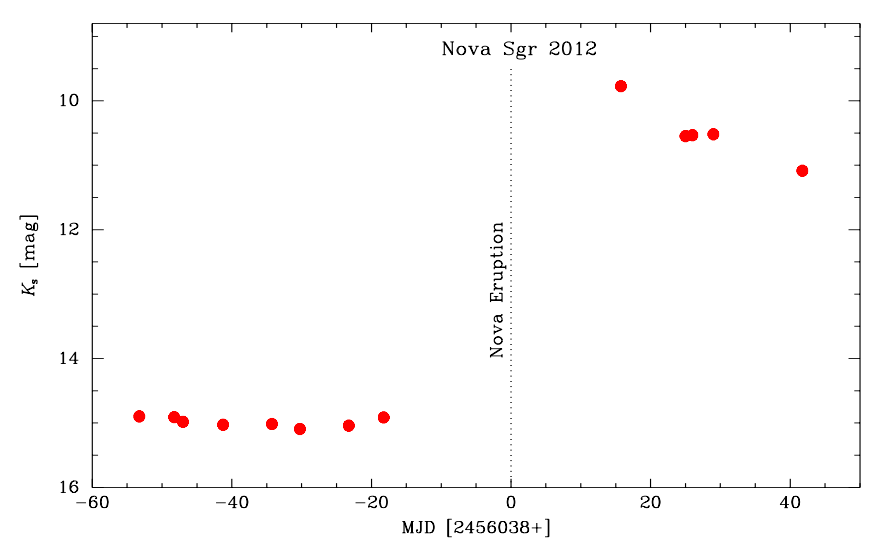

Fig. 8. VVV $K_{\mathrm{s}}$-band light curve of Nova Sgr 2012b. Its eruption was detected on $21 \mathrm{Apr}$. 2012. The nova progenitor is seen at $K_{\mathrm{s}} \sim 15.0 \mathrm{mag}$ in five observations earlier in 2012, while a few observations starting about a hundred days after the eruption registered its fading curve.

material seems in fact to suggest for the ejecta of classical novae a lower mass and higher velocities $\left(10^{-4}-10^{-5} M_{\odot}\right.$ and up to $6000 \mathrm{~km} \mathrm{~s}^{-1}$, respectively, as in the extreme case of LMC1991 Schwarz et al. 2001) than thought before. Identifying where such a discrepancy stems from would have dramatic consequences on our understanding of the nova phenomenon, e.g., by clarifying its role in the chemical evolution of the galaxy.

By supposing expansion velocities of the nova remnants larger than $1000 \mathrm{~km} \mathrm{~s}^{-1}$, it is reasonable to expect that at least the closest/fastest novae produce nebular remnants that eventually may be spatially resolved even from ground-based telescopes. Imaging such remnants allows the investigation of fundamental correlations between the properties of the central binary, the evolution of the outburst and the ejecta shaping mechanism, and also provides a direct measure of the distance to the nova by combining the angular expansion rate with spectroscopically derived expansion velocities, i.e., the so-called expansion parallax method (Baade 1940; Martin 1989).

While the infrared imagery has several advantages over the optical one (e.g., the extinction - which is often poorly known for novae - is conveniently reduced), up to now only a handful of nova remnants have been searched (and even fewer spatially resolved) in this spectral region, compared to more than 40 nova remnants resolved in the optical and $\sim 10$ in the radio.
Thanks to the large sky area covered and to the higher spatial resolution than previous IR surveys (such as 2MASS and DENIS), VVV is in unique position to perform for the first time a systematic search for remnants of classical novae that exploded in the last decades within the inner, highly-extincted regions of the MW. Even assuming a representative distance to a nova of $4 \mathrm{kpc}$ (about half-way to the Galactic centre) and a very conservative expansion velocity of $1000 \mathrm{~km} \mathrm{~s}^{-1}$, VVV is in fact potentially able to spatially resolve the corresponding nebular remnants after $\sim 40$ years from the outburst, taking into account the VIRCAM pixel scale $\left(0.34\right.$ pixel $\left.^{-1}\right)$ and a nebula angular size of $2^{\prime \prime}$. No resolved novae shells have been detected yet in the VVV imaging data. In a forthcoming phase of the survey, a search for novae using imaging will be conducted on the deep, stacked $K_{\mathrm{s}}$-band images, using more focused image analysis such as point spread function (PSF) subtraction or difference image analysis (DIA, e.g., Saito et al. 2012a).

\section{Conclusions}

We have presented a near-IR catalogue of novae within the VVV area covering the MW bulge and southern Galactic plane area. From the 140 objects found in the VSX/AAVSO catalogue, we reported the $J H K_{\mathrm{s}}$ colours of 93 objects. The rest were beyond detection or were heavily blended sources. Colour-magnitude and colour-colour diagrams were presented, as well as CMDs using dereddened colours and reddening-free indices. These should be used with caution, since in the case of nearby objects, the dereddened colours can overestimate the corrections, by assuming the total extinction in the line of sight. Likewise, reddening-free near-IR indices specifically devised for studying of novae would also be of considerable interest, given their different spectral shapes compared with those typically assumed in computing these indices.

Thanks to its higher spatial resolution in the near-IR and wide $K_{\mathrm{s}}$-range covered by the observations, the VVV survey can be a major contributor in the search for and study of novae in the Galaxy, mainly in the most crowded and high-extinction regions of the MW, beyond the capabilities of the current searches for novae in optical wavelengths.

VVV can produce well-sampled light curves covering many years for Galactic novae belonging both to the bulge and the southern part of the disk, even for objects during or fading after eruption. The recent report of VVV-NOV-001, the first nova candidate from the VVV data discovered in the inner bulge, is an illustrative example. Surely, the first of many more to come. The possibility to search for novae using the VVV imaging is accordingly a very promising path to unveiling the heretofore hidden population of heavily obscured novae.

Acknowledgements. We gratefully acknowledge use of data from the ESO Public Survey programme ID 179.B-2002 taken with the VISTA telescope, data products from the Cambridge Astronomical Survey Unit, and funding from the FONDAP Center for Astrophysics 15010003, the BASAL CATA Center for Astrophysics and Associated Technologies PFB-06, the FONDECYT from CONICYT, and the Ministry for the Economy, Development, and Tourism's Programa Iniciativa Científica Milenio through grant P07-021-F, awarded to The Milky Way Millennium Nucleus. Support for R.A. is provided by Proyecto GEMINI CONICYT 32100022 and by a Postdoctoral Fellowship from the School of Engineering at Pontificia Universidad Católica de Chile. D.M. acknowledges funding from Proyecto FONDECYT Regular 1130196. J.C.B. acknowledges support from a Ph.D. Fellowship from CONICYT. M.C. and I.D. acknowledge funding from Proyecto FONDECYT Regular 1110326. J.B. acknowledges funding from Proyecto FONDECYT Regular 1120601. R.K. acknowledges partial support from FONDECYT through grant no. 1130140. This research made use of the International Variable Star indeX (VSX) database, operated at the AAVSO, Cambridge, Massachusetts, USA. 
R. K. Saito et al.: A near-IR catalogue of the Galactic novae in the VVV survey area

\section{Appendix A: VVV Novae finding charts}

In this section we present the finding charts for all Novae in the VVV Survey area, listed in Table 1 . In most cases the charts are $J H K_{\mathrm{s}}$ composite images, unless when explicitly marked as a $K_{\mathrm{s}}$-band image. All charts are $30^{\prime \prime} \times 30^{\prime \prime}$ in size, oriented in Galactic coordinates, with the positive Galactic longitude pointing up. A cross marks the coordinates given by the catalogue and helps one to check the notes we adopted in Table 1 .

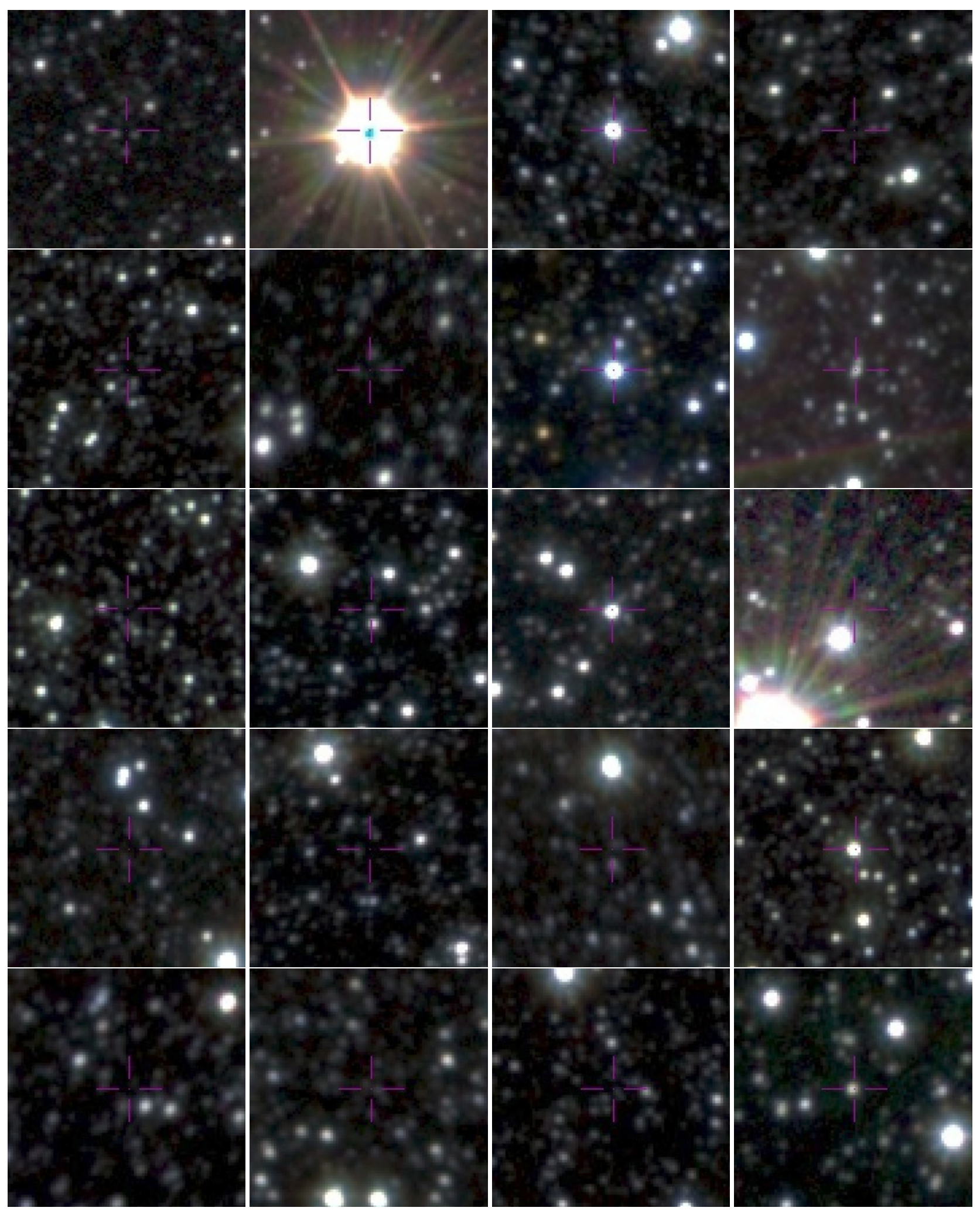

Fig. A.1. First row: Nova Sgr 1893, Nova Sgr 1897, Nova Sgr 1899 and Nova Sco 1901. Second row: Nova Sgr 1901, Nova Sgr 1905, Nova Cir 1906 and Nova Sco 1906. Third row: Nova Sgr 1910, Nova Sgr 1914c, Nova Sgr 1917 and Nova Sgr 1919. Fourth row: Nova Sco 1922 , Nova Sgr 1924, Nova Sgr 1926, Nova Sgr 1926a. Last row: Nova Sco 1928, Nova Sgr 1928, Nova Sgr 1930 and Nova Cen 1931. 


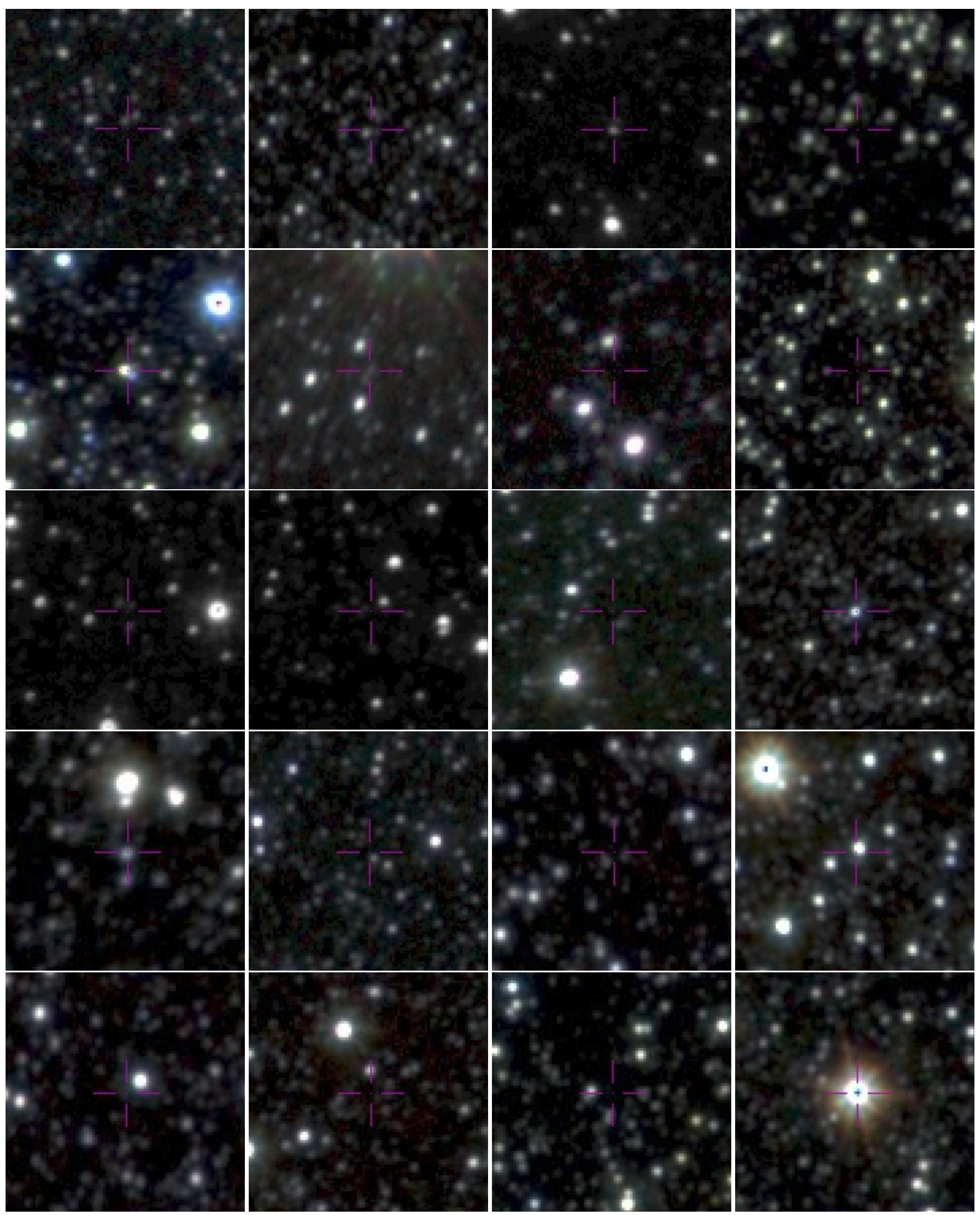

Fig. A.1. continued. First row: Nova Sgr 1932, Nova Sgr 1933, Nova Cru 1935 ( $K_{\mathrm{s}}$-band image) and Nova Sco 1935. Second row: Nova Sgr 1936, Nova Sgr 1936b, Nova Sgr 1936c and Nova Sgr 1936d. Third row: Nova Sgr 1937 ( $K_{\mathrm{s}}$-band image), Nova Oph 1940 ( $K_{\mathrm{s}}$-band image), Nova Sco 1941 and Nova Sgr 1943. Fourth row: Nova Sco 1944, Nova Sgr 1944, Nova Sgr 1945a and Nova Sgr 1945b. Last row: Nova Sgr 1947 a, Nova Sgr 1948, Nova Sco 1949 and Nova Oph 1950. 
R. K. Saito et al.: A near-IR catalogue of the Galactic novae in the VVV survey area

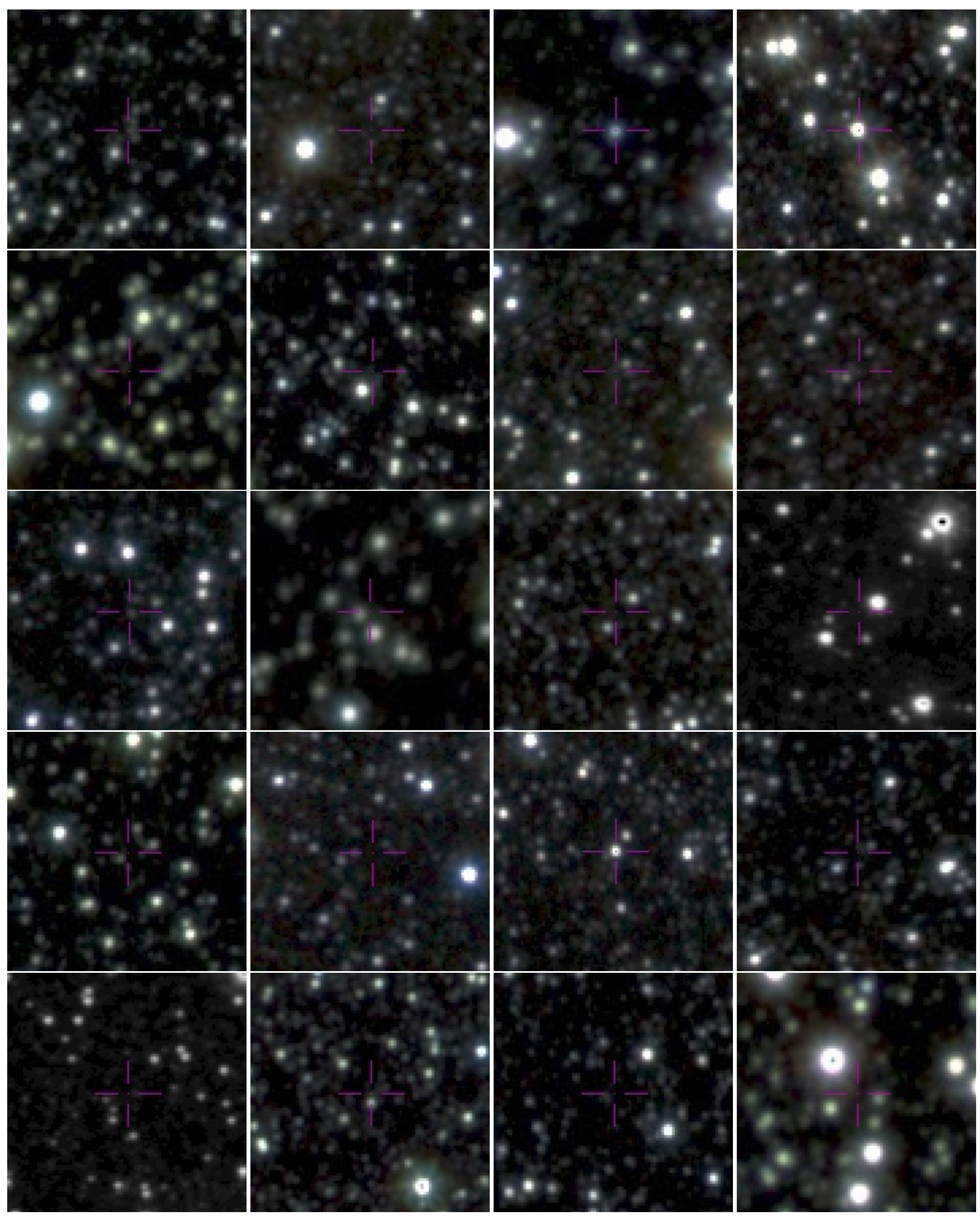

Fig. A.1. continued. First row: Nova Sco 1950a, Nova Sco 1950b, Nova Sco 1950c and Nova Sgr 1951a. Second row: Nova Sgr 1951b, Nova Sco 1952, Nova Sco 1952a and Nova Sco 1952b. Third row: Nova Sgr 1952a, Nova Sgr 1952b, Nova Sgr 1953 and Nova Oph 1954 (K $K_{\mathrm{s}}$-band image). Fourth row: Nova Sco 1954, Nova Sgr 1954a, Nova Sgr 1954b and Nova Sgr 1955. Last row: Nova Oph 1957 (K s $_{\mathrm{s}}$ band image), Nova Sgr 1960, Nova Oph 1961 and Nova Sgr 1963. 




Fig. A.1. continued. First row: Nova Sco 1964, Nova Sgr 1968, Nova Sgr 1974 and Nova Sgr 1975. Second row: Nova Sgr 1977, Nova Sgr 1978, Nova Sgr 1980 and Nova Sgr 1982. Third row: Nova Nor 1983, Nova Oph 1983, Nova Sgr 1983 and Nova Sgr 1984. Fourth row: Nova Sco 1985, Nova Sgr 1986, Nova Sgr 1987 and Nova Sco 1989b. Last row: Nova Cen 1991, Nova Oph 1991b, Nova Sgr 1991 and Nova Sco 1992. 
R. K. Saito et al.: A near-IR catalogue of the Galactic novae in the VVV survey area

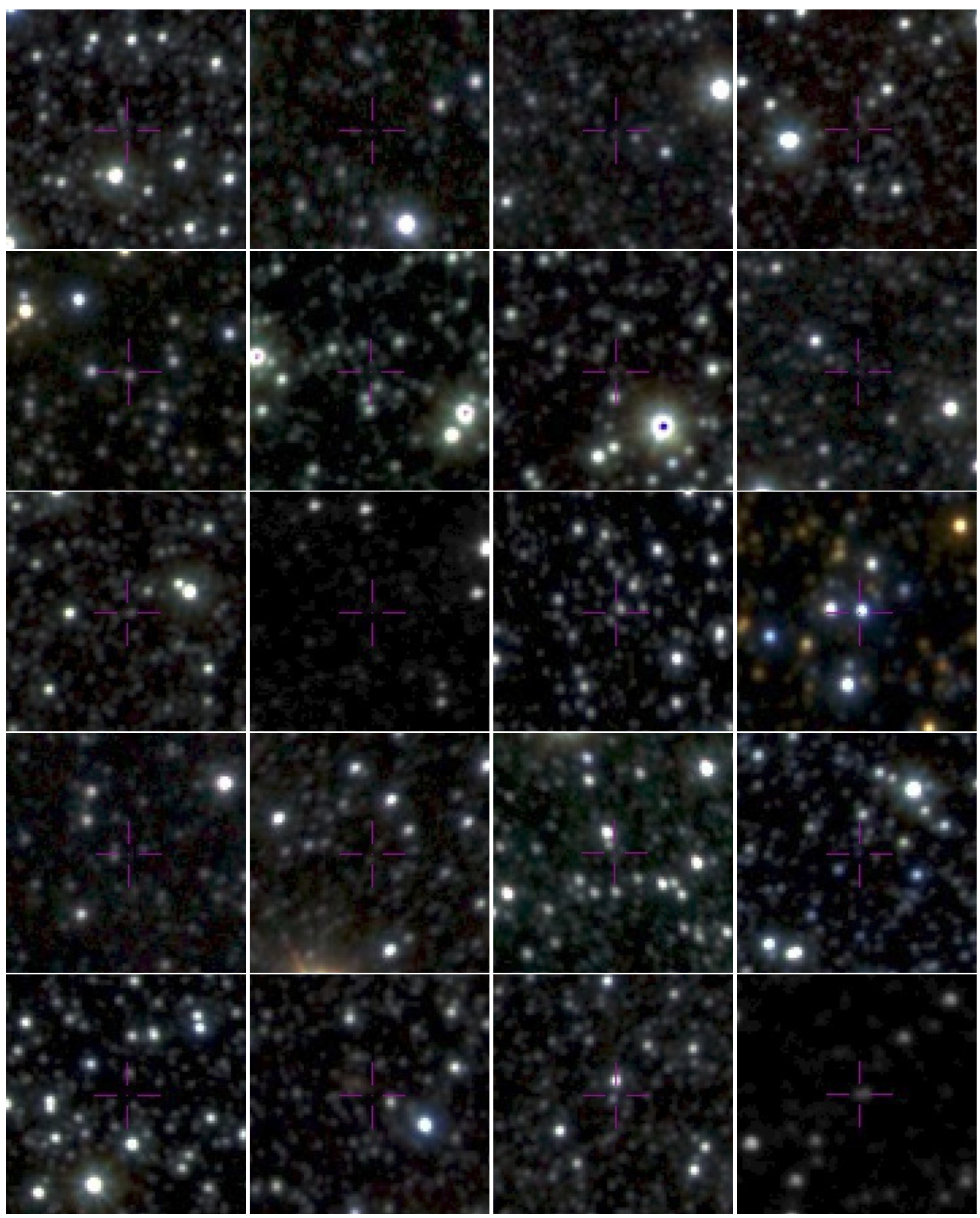

Fig. A.1. continued. First row: Nova Sgr 1992a, Nova Sgr 1992b, Nova Sgr 1992c and Nova Sgr 1993. Second row: Nova Cru 1996, Nova Sco 1997, Nova Sco 1998 and Nova Sgr 1998. Third row: Nova Sgr 1999, Nova Sgr 2000 ( $K_{\mathrm{s}}$-band image), Nova Sco 2001 and Nova Sgr 2001. Fourth row: Nova Sgr 2001b, Nova Sgr 2001c, Nova Sgr 2002 and Nova Sgr 2002b. Last row: Nova Sgr 2002d, Nova Sgr 2003 b, Nova Oph 2004 and Nova Sco 2004 ( $K_{\mathrm{s}}$-band image). 




Fig. A.1. continued. First row: Nova Sco 2004b, Nova Sgr 2004, Nova Cen 2005 and Nova Nor 2005. Second row: Nova Sco 2005, Nova Sgr 2005a, Nova Sgr 2005b and Nova Oph 2006. Third row: Nova Sgr 2006, Nova Oph 2007, Nova Nor 2007 and Nova Oph 2008a. Fourth row: Nova Oph 2008b ( $K_{\mathrm{s}}$-band image), Nova Sgr 2008, Nova Sgr 2008b and Nova Oph 2009. Last row: Nova Sgr 2009a, Nova Sgr 2009b, Nova Sgr 2009c and Nova Oph 2010. 
R. K. Saito et al.: A near-IR catalogue of the Galactic novae in the VVV survey area

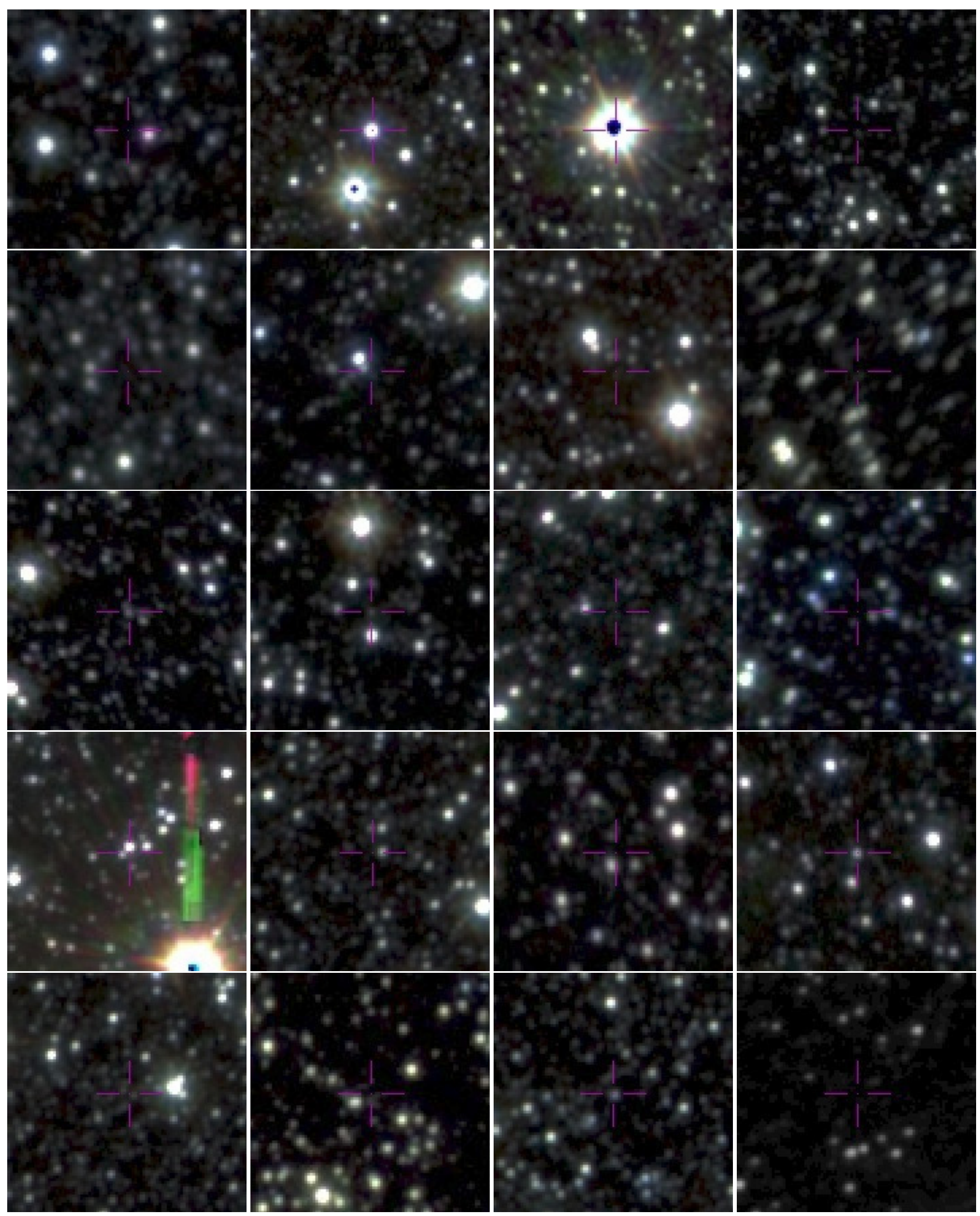

Fig. A.1. continued. First row: Nova Oph 2010b, Nova Sgr 2010, Nova Sgr 2010b and Nova Sgr 2011. Second row: Nova Sgr 2011b, Nova Cen 2012, Nova Oph 2012b and Nova Sco 2012. Third row: Nova Sgr 2012, Nova Sgt 2012c, Nova Sgt 2012d and OGLE-NOVA-001. Fourth row: V1213 Cen, PN G002.6, V0733 Sco and GR Sgr. Last row: AT Sgr, V2859 Sco, KW2003 105 and V0729 Sco. 


\section{References}

Ashok, N. M., Banerjee, D. P. K., Varricatt, W. P., \& Kamath, U. S. 2006, MNRAS, 368, 592

Baade, W. 1940, PASP, 52, 386

Banerjee, D. P. K., \& Ashok, N. M. 2012, Bull. Astron. Soc. India, 40, 243

Cardelli, J. A., Clayton, G. C., \& Mathis, J. S. 1989, ApJ, 345, 245

Catelan, M., Minniti, D., Lucas, P. W., et al. 2011, in RR Lyrae Stars, Metal-Poor Stars, and the Galaxy, 145

Chen, B. Q., Schultheis, M., Jiang, B. W., et al. 2013, A\&A, 550, A42

Darnley, M. J., Bode, M. F., Kerins, E., et al. 2004, MNRAS, 353, 571

Darnley, M. J., Bode, M. F., Kerins, E., et al. 2006, MNRAS, 369, 257

della Valle, M., \& Livio, M. 1994, A\&A, 286, 786

della Valle, M., \& Livio, M. 1998, ApJ, 506, 818

Dobrotka, A., Retter, A., \& Liu, A. 2008, A\&A, 478, 815

Ducati, J. R., Bevilacqua, C. M., Rembold, S. B., \& Ribeiro, D. 2001, ApJ, 558, 309

Epchtein, N., de Batz, B., Copet, E., et al. 1994, Ap\&SS, 217, 3

Frank, J., King, A., \& Raine, D. J. 2002, Accretion Power in Astrophysics (Cambridge, UK: Cambridge University Press), 398

Gaposchkin, C. H. P. 1957, The galactic novae, Amsterdam, North-Holland Pub. Co. (New York: Interscience Publishers)

Green, D. W. E. 2012a, Central Bureau Electronic Telegrams, 3073, 1

Green, D. W. E. 2012b, Central Bureau Electronic Telegrams, 3089, 1

Greenhouse, M. A., Grasdalen, G. L., Hayward, T. L., Gehrz, R. D., \& Jones, T. J. 1988, AJ, 95, 172

Gonzalez, O. A., Rejkuba, M., Zoccali, M., et al. 2012, A\&A, 543, A13

Liller, W. 1993, Mex. Astron. Astrofis, 26, 41

Liller, W., \& Mayer, B. 1987, PASP, 99, 606

Martin, P. G. 1989, in Classical Novae, eds. M. F. Bode, \& A. Evans (Oxford: Wiley), 73

Mason, C. G., Gehrz, R. D., Woodward, C. E., et al. 1998, ApJ, 494, 783

Minniti, D., Lucas, P. W., Emerson, J. P., et al. 2010, New Astron., 15, 433

Nakano, S., Nishiyama, K., \& Kabashima, F. 2008, Central Bureau Electronic Telegrams, 1342, 1

Nakano, S., Nishimura, H., Itagaki, K., et al. 2010a, IAU Circ., 9111, 2

Nakano, S., Nishimura, H., Kiyota, S., et al. 2010b, IAU Circ., 9119, 1

Nakano, S., Nishimura, H., Kiyota, S., \& Yusa, T. 2011, IAU Circ., 9196, 1

Nishimura, H., \& Nakano, S. 2012, Central Bureau Electronic Telegrams, 3166, 1

Nishiyama, S., Tamura, M., Hatano, H., et al. 2009, ApJ, 696, 1407
Nishiyama, K., Kabashima, F., Liller, W., Yusa, T., \& Maehara, H. 2010, IAU Circ., 9140, 1

Nishiyama, K., Kabashima, F., Maehara, H., \& Kiyota, S. 2011, IAU Circ., 9203, 1

Kiyota, S., Vollmann, W., Koberger, H., et al. 2010, IAU Circ., 9112, 1

Kozlowski, S., Poleski, R., Udalski, A., et al. 2012, The Astronomer's Telegram, 4323, 1

Raj, A., Ashok, N. M., \& Banerjee, D. P. K. 2011, MNRAS, 415, 3455

Rieke, G. H., \& Lebofsky, M. J. 1985, ApJ, 288, 618

Russell, R. W., Rudy, R. J., Lynch, D. K., et al. 2008, IAU Circ., 8948, 1

Saito, R. K., \& Minniti, D. 2012a, The Astronomer's Telegram, 4353, 1

Saito, R. K., \& Minniti, D. 2012b, The Astronomer's Telegram, 4372, 1

Saito, R. K., Hempel, M., Minniti, D., et al. 2012a, A\&A, 537, A107

Saito, R. K., Minniti, D., Dias, B., et al. 2012b, A\&A, 544, A147

Saito, R. K., Minniti, D., Angeloni, R., \& Catelan, M. 2012c, The Astronomer's Telegram, 4426, 1

Sala, G., Hernanz, M., Ferri, C., \& Greiner, J. 2008, ApJ, 675, L93

Sala, G., Hernanz, M., Ferri, C., \& Greiner, J. 2010, Astron. Nachr., 331, 201

Sato, S., Kawara, K., Kobayashi, Y., et al. 1978, PASJ, 30, 419

Schwarz, G. J., Shore, S. N., Starrfield, S., et al. 2001, MNRAS, 320, 103

Shafter, A. W. 1997, ApJ, 487, 226

Shafter, A. W., \& Irby, B. K. 2001, ApJ, 563, 749

Shafter, A. W., Bode, M. F., Darnley, M. J., et al. 2011, ApJ, 727, 50

Shara, M. M., Yaron, O., Prialnik, D., \& Kovetz, A. 2010, ApJ, 712, L143

Shaviv, N. J. 2002, Classical Nova Explosions, 637, 259

Shaviv, N. J., \& Dotan, C. 2012, Mem. Soc. Astron. It., 83, 792

Skrutskie, M. F., Cutri, R. M., Stiening, R., et al. 2006, AJ, 131, 1163

Sun, G., \& Gao, X. 2009, IAU Circ., 9049, 1

Varricatt, W. P., Banerjee, D. P. K., \& Ashok, N. M. 2012, The Astronomer's Telegram, 4405, 1

Venturini, C. C., Rudy, R. J., Lynch, D. K., Mazuk, S., \& Puetter, R. C. 2002, AJ, 124, 3009

Waagen, E. O. 2012, AAVSO Alert Notice, 461, 1

Walter, F. M., \& Buil, C. 2012, Central Bureau Electronic Telegrams, 3124, 1

Warner, B. 2003, in Cataclysmic Variable Stars (Cambridge, UK: Cambridge University Press), 592

Williams, R. E. 1992, AJ, 104, 725

Woodward, C. E., Gehrz, R. D., Jones, T. J., Lawrence, G. F., \& Skrutskie, M. F. 1997, ApJ, 477, 817

Yamaoka, H., \& Itagaki, K. 2012, Central Bureau Electronic Telegrams, 3156, 1 